
454, 154-157; 2008) and the accompanying Editorial 'An unnecessary battle' (Nature 454, 137-138; 2008).

Mental health: maybe human troubles don't fit into set categories

SIR - We would like to suggest possible reason for the disputes and failures in the search for a genetic basis for mental illnesses that was not mentioned in your News Feature and Editorial. It is that the discrete categories defined by the American Psychiatric Association's fourth Diagnostic and Statistical Manual of Mental Disorders (DSM) checklists are essentially arbitrary, rarely or never corresponding to a particular and consistent underlying biological dysfunction.

The DSM identifies human troubles as patterns of feelings and behaviours, given in generic verbal descriptions and grouped together into categories defined by checklists. The feelings and behaviours figuring on a particular checklist are described as

"symptoms" of the condition that the checklist supposedly identifies.

A symptom is the manifestation of a particular disease state, but the DSM checklists rarely capture any such thing. The diagnostic categories are complex, vague and subjective. There is considerable overlap between conditions and inconsistency within them, both in 'symptoms' and 'treatments'. Their character is entirely generic, failing to incorporate personal histories of patients into a description of their situation. And they are cut from whole cloth by committees whose members have an inherent professional interest in drawing human difficulties into their purview as clinicians and researchers. The well known interconnections between scientific and commercial outcomes in this area are also problematic.

Rather than identifying discrete and universal biological disease phenomena, then, DSM categories may best be viewed as expedient, arbitrary distinctions, used to justify psychiatry's claims to scientific legitimacy and to simplify the task of gaining approval for standardized medical responses to complex human problems.

Assuming that all variations in human experience and character are mediated in part by variations in body chemistry, biological correlates for these groupings of feelings and behaviours can presumably be turned up with enough searching. The associated problems may nonetheless be better understood and dealt with in the context of human lives, relationships, societies and environments, without depending on such categorization.

Contemporary psychiatry is in far too deep to accept such a challenge easily. However, that should not prevent us from considering it.

Paul Reeve Laboratoire Psychologie de la Perception, 45 rue des Saints Pères, 75006 Paris, France e-mail: paul.reeve@mail.mcgill.ca Louigi Addario-Berry Department of Statistics, University of Oxford, 1 South Parks Road, Oxford OX13TG, UK

\section{Mental health: don't overlook environment and its risk factors}

SIR - You highlight the need to adopt a more integrated perspective when trying to unravel the biological complexity of neuropsychiatric disorders such as schizophrenia and depression. But the 'battle' between genetics and neuroscience, despite being well funded, may be missing the point.

Napoleon Bonaparte advised:

"Never interrupt your enemy when he is making a mistake."

Those of us who assess the contribution of non-heritable risk factors to neuropsychiatric illness would like to politely interrupt this battle to remind opponents that environmental risk factors have now overtaken genetic factors with respect to both effect size and the proportion of the population that is affected.

For schizophrenia, for example, factors relating to urban birth, cannabis use and migrant status are well replicated and have relatively large effects - in contrast to the scant evidence that remains after decades of genetics research. Although the 'heritability index' for schizophrenia is large (about $85 \%$ ), this metric encompasses the neglected contribution of gene-environment interactions, as well as the high-profile genetic component. This key point is largely forgotten in the heat of the battle.

It has been convincingly argued (A. Caspi and T. E. Moffitt Nature Rev. Neurosci. 7, 583-590; 2006) that the power to detect genuine genetic-susceptibility loci would be substantially increased if we could stratify samples according to environmental risk factors. Let's have more funding to help fine-map the wide range of nonheritable risk factors associated with disabling disorders such as schizophrenia and depression, and discover how they act. These clues are too valuable to overlook. John J. McGrath Queensland Centre for Mental Health Research, University of Queensland, St Lucia, Queensland 4076, Australia e-mail:john_mcgrath@qcmhr.uq.edu.au Jean-Paul Selten Department of Psychiatry, University Medical Centre, Utrecht 3508 GA, The Netherlands

\section{Mental health: drop ideological baggage in favour of best tools}

SIR - Your News Feature summarizes the problems in the hunt for genetic variants associated with psychiatric disorders. But it contains ambiguities that, coupled with strong opinions from leading geneticists and neuroscientists, leave an unnecessarily dire impression of the field.

The News Feature gives DISC1

('disrupted-in-schizophrenia 1') as a prototypical example of a gene bearing variants that fail to manifest consistently in the clinic - something that often plagues psychiatric genetics. As you imply, these failures are at odds with the clear association of certain genetic variants and rare mutations with an increased risk of a particular disease, such as Alzheimer's.

However, selective pressures may prevent mutations associated with strong deleterious effects from spreading into more common variants. The failure of large-scale, genome-wide and candidate-gene association studies to reliably detect common risk variants in DISC1 does not diminish the significance of the rare DISC1 structural mutation found in the Scottish family mentioned in the News Feature, nor the possibility of other rare DISC1 mutations affecting disease risk. Keeping an open mind is important, given increasing evidence that rare structural mutations play a major role in the aetiology of psychiatric disorders (see Nature doi:10.1038/ news.2008.994; 2008).

Efforts are under way to combine the best approaches from genetics and neuroscience. Genome-wide association studies for intermediate phenotypes are being linked with psychiatric disorders, circumventing the bias inherent in candidate-gene investigations and the questionable validity of current diagnostic labels (R. M. Bilder Biol. Psychiat. 63, 439-440; 2008).

I hope that today's students and young researchers will not inherit the ideological baggage of their fields, but rather will be armed with the tools needed to tackle specific problems, regardless of departmental or disciplinary borders.

P. Alexander Arguello Columbia University, 1051 Riverside Drive, New York, New York 10032, USA e-mail: paa2102@columbia.edu 